\title{
Transcranial photoacoustic tomography of the monkey brain
}

Liming Nie, Chao Huang, Zijian Guo, Mark Anastasio, Lihong V. Wang

Liming Nie, Chao Huang, Zijian Guo, Mark Anastasio, Lihong V. Wang, "Transcranial photoacoustic tomography of the monkey brain," Proc. SPIE 8223, Photons Plus Ultrasound: Imaging and Sensing 2012, 82230L (23 February 2012); doi: 10.1117/12.907060

SPIE. Event: SPIE BiOS, 2012, San Francisco, California, United States 


\title{
Transcranial photoacoustic tomography of the monkey brain
}

\author{
Liming Nie ${ }^{\text {a) }}$, Chao Huang ${ }^{\text {a) }}$, Zijian Guo, Mark Anastasio*, and Lihong V. Wang* \\ Dept. of Biomedical Engineering, Washington University in St. Louis, 1 Brookings Dr., St. Louis, \\ MO, USA 63130 \\ *Corresponding authors: anastasio@seas.wustl.edu lhwang@biomed.wustl.edu
}

\begin{abstract}
A photoacoustic tomography (PAT) system using a virtual point ultrasonic transducer was developed for transcranial imaging of monkey brains. The virtual point transducer provided a 10 times greater field-of-view (FOV) than finiteaperture unfocused transducers, which enables large primate imaging. The cerebral cortex of a monkey brain was accurately mapped transcranially, through up to two skulls ranging from 4 to $8 \mathrm{~mm}$ in thickness. The mass density and speed of sound distributions of the skull were estimated from adjunct X-ray CT image data and utilized with a timereversal algorithm to mitigate artifacts in the reconstructed image due to acoustic aberration. The oxygenation saturation $\left(\mathrm{sO}_{2}\right)$ in blood phantoms through a monkey skull was also imaged and quantified, with results consistent with measurements by a gas analyzer. The oxygenation saturation $\left(\mathrm{sO}_{2}\right)$ in blood phantoms through a monkey skull was also imaged and quantified, with results consistent with measurements by a gas analyzer. Our experimental results demonstrate that PAT can overcome the optical and ultrasound attenuation of a relatively thick skull, and the imaging aberration caused by skull can be corrected to a great extent.
\end{abstract}

Keywords: Transcranial photoacoustic tomography, time-reversal image reconstruction

\section{INTRODUCTION}

Photoacoustic tomography (PAT) is a noninvasive hybrid imaging modality that possesses strong optical absorption contrast and high ultrasound spatial resolution [1,2]. It relies on the thermoelastic effect for generating acoustic pressure waves under thermal and stress confinement conditions. A pulsed laser is used to irradiate tissue, causing optical absorption, rapid thermoelastic expansion, and acoustic pressure generation [3]. When the excitation laser is replaced by a microwave source, the technique is named thermoacoustic tomography (TAT) [4-6]. PAT has been applied for in vivo imaging of vascular structural and hemodynamic functional imaging [7-8]. Structural and functional imaging of the mouse brain has been well demonstrated with PAT $[9,10]$.

Traditional flat transducers can provide only a small field-of-view (FOV) with acceptable sensitivity; thus PAT and TAT of the cerebral cortex in large animals $[11,12]$ is challenging. There is always a compromise between sensitivity and the detecting angle: large-aperture detectors can achieve better sensitivity while compromising the FOV, whereas small point detectors produce a larger FOV while compromising the detection sensitivity. Negative lens transducers introduce attenuation and reverberation of ultrasonic waves, which decrease sensitivity and degrade reconstruction accuracy. The negative-lens transducers were designed primarily for conceptual demonstration, and are not suitable for robust animal imaging [13].

Transcranial brain imaging represents an important application that may benefit significantly by the advancement of PAT methods. PAT detects ultrasound signals through one-way propagation, and thus suffers much less distortion and attenuation by the skull than pulse echo does. In contrast to ultrasound imaging, PAT detects ultrasound signals through one-way propagation, and thus suffers much less distortion and attenuation by the skull. In addition, PAT based on endogenous hemoglobin contrast is particularly suitable for imaging the blood vessels of the cerebral cortex. Multi-wavelength PAT can be used to compute oxygen saturation of hemoglobin and to monitor functional activities of the human cerebral cortex.

Photons Plus Ultrasound: Imaging and Sensing 2012, edited by Alexander A. Oraevsky, Lihong V. Wang, Proc. of SPIE Vol. 8223, 82230L · (C) 2012 SPIE · CCC code: 1605-7422/12/\$18 · doi: 10.1117/12.907060 
In this study, we investigate transcranial PAT imaging of monkey brains. The PAT system possesses a large FOV due to the use of a custom built virtual point ultrasonic detector. In comparison to the negative-lens ultrasonic transducers, the custom-built detector retains a large acceptance angle, provides a higher SNR, and avoids ultrasound reverberations. Consequentially, the shapes of the imaging objects far away from the scanning center were preserved with both uniform high spatial resolution and SNR. We demonstrate that a high uniformity in resolution and can be achieved within a FOV of $6 \mathrm{~cm}$ diameter, even when imaging through a monkey skull. The cerebral cortex of a monkey brain was accurately mapped transcranially, through one or even two skull thicknesses ranging from 4 to $8 \mathrm{~mm}$. To render PAT an effective modality for transcranial imaging in large primates, including humans, we propose a reconstruction methodology for transcranial PAT to mitigate skull-induced aberration in the reconstructed image. Our experimental results show that the reconstruction methodology can reduce artifact levels as compared to a previously employed back-projection algorithm.

\section{RECONSTRUCTION METHODOLOGY}

The goal of PAT image reconstruction is to obtain an estimate of the laser-inducted pressure wavefiled $p_{0}$ from the measured pressure wavefield data $\left\{\hat{p}\left(r_{m}, t\right)\right\}_{m=1}^{M}$, where $r_{m}$ denotes the location of the $\mathrm{m}$-th ultrasonic transducer, and $\mathrm{M}$ indicates the number of measurement locations. To reconstruct PAT images in heterogeneous media, we utilized a kspace time-reversal algorithm [14] for image reconstruction, which can compensate for the distortion introduced into the measurement data by heterogeneities. When acoustic attenuation and shear wave mode-conversion are neglected, the time-reversal reconstruction algorithm operates by iteratively solving the discretized versions of following three coupled equations backward in time:

with initial and boundary conditions specified as:

$$
\begin{gathered}
\frac{\partial}{\partial t} u(\vec{r}, t)=-\frac{1}{\rho_{0}} p(\vec{r}, t) \\
\frac{\partial}{\partial t} \rho(\vec{r}, t)=-\rho_{0}(\vec{r}) \nabla \cdot u(\vec{r}, t) \\
p(\vec{r}, t)=c_{0}(\vec{r})^{2} \rho(\vec{r}, t),
\end{gathered}
$$

$$
\left.p(\vec{r}, t)\right|_{t=T}=0,\left.\quad u(\vec{r}, t)\right|_{t=T}=0, p\left(r_{m}, t\right)=\hat{p}\left(r_{m}, t\right) .
$$

Here, $p(\vec{r}, t)$ is the pressure wavefield, $u(\vec{r}, t)$ is the acoustic particle velocity, $T$ denotes the maximum time at which the pressure data were recorded, $c_{0}(\vec{r})$ denotes the medium's speed of sound (SOS) distribution, and $\rho(\vec{r}, t)$ and $\rho_{0}(\vec{r})$ describe the distributions of the medium's acoustic and ambient densities, respectively. The time-reversal algorithm is based on a full-wave solution to the acoustic wave equation for heterogeneous media; therefore it can compensate for the aberration in the photoacoustic signals due to the variations in $c_{0}(\vec{r})$ and $\rho_{0}(\vec{r})$ within the skull.

The SOS and mass density distribution of the skull can be estimated from a porosity map $\Phi_{k}$, which is given by:

$$
\Phi_{k}=1-\frac{H_{k}}{H_{k}^{\text {Max }}}
$$

where $H_{k}$ denotes the value (Hounsfield Units) of the k-th voxel in the X-ray CT image, and $H_{k}^{\text {Max }}$ is the maximum value of $H_{k}$.

The mass density map $\rho_{k}$ can be estimated from the porosity map as

$$
\rho_{k}=\Phi_{k} \rho^{w}+\left(1-\Phi_{k}\right) \rho^{s},
$$

where $\rho^{w}=1000 \mathrm{~kg} / \mathrm{m}^{3}$ is the density of water, and $\rho^{s}=2100 \mathrm{~kg} / \mathrm{m}^{3}$ is the maximum mass density in the skull bone $[15,16]$.

The SOS map $c_{k}$ can also be determined from the porosity map as

$$
c_{k}=\Phi_{k} c^{w}+\left(1-\Phi_{k}\right) c^{s},
$$

where $c^{w}=1480 \mathrm{~kg} / \mathrm{m}^{3}$ is the SOS in water, and $c^{s}=2900 \mathrm{~kg} / \mathrm{m}^{3}$ is the maximum SOS in the skull bone $[15,16]$.

\section{METHODS}

A PAT system was employed for monkey brain imaging, as shown in Fig. 1(a). A Q-switched Nd:YAG laser (PRO-350- 
10 , Newport) with a $6.5 \mathrm{~ns}$ pulse duration and a $10 \mathrm{~Hz}$ pulse repetition rate was used as an energy source. The laser beam was expanded by use of a piece of concave lens and homogenized by a piece of ground glass before illuminating the target. The energy density of the laser beam on the skull was controlled to $8 \mathrm{~mJ} / \mathrm{cm}^{2}$ (within the ANSI standard) [17]. The target sample was immersed in water and irradiated by a laser beam from the top, and an ultrasound detector scanned the sample circularly with a scanning radius of $9 \mathrm{~cm}$ on the plane orthogonal to the irradiation direction over 400 angles. The PA signals received by the transducer were amplified by a $50-\mathrm{dB}$ amplifier (5072 PR, Panametrics, Waltham, MA), then directed to a data-acquisition (DAQ) card (Compuscope 14200; Gage Applied, Lockport, IL). The DAQ card was triggered by the Q-switch signal from the laser to acquire the PA signals simultaneously. The DAQ card features a high-speed 14-bit analog-to-digital converter with a sampling rate of $50 \mathrm{MS} / \mathrm{s}$. The raw data transferred by the DAQ card was then stored in the PC for imaging reconstruction.

The custom-built virtual point ultrasonic detector (from GE Healthcare) employed had a $2.25-\mathrm{MHz}$ central frequency with $70 \%$ bandwidth. The virtual detector is equivalent to point detector in their receiving angle and FOV; however, it possesses a much greater receiving surface and sensitivity. As shown in Fig. 1(b), the virtual point of the convex transducer is located behind the receiving surface. The focus is $9.7 \mathrm{~mm}$ behind the most protruding point on the transducer surface. The acceptance angle is defined as the angle at which the radiation power decreases more than $6 \mathrm{~dB}$. The convex part of the curved surface is $2.5 \mathrm{~mm}$ in depth, and its cross sectional diameter is $13 \mathrm{~mm}$. Thus the receiving angle is $87.8^{\circ}$, compared to $8.6^{\circ}$ for a flat transducer.
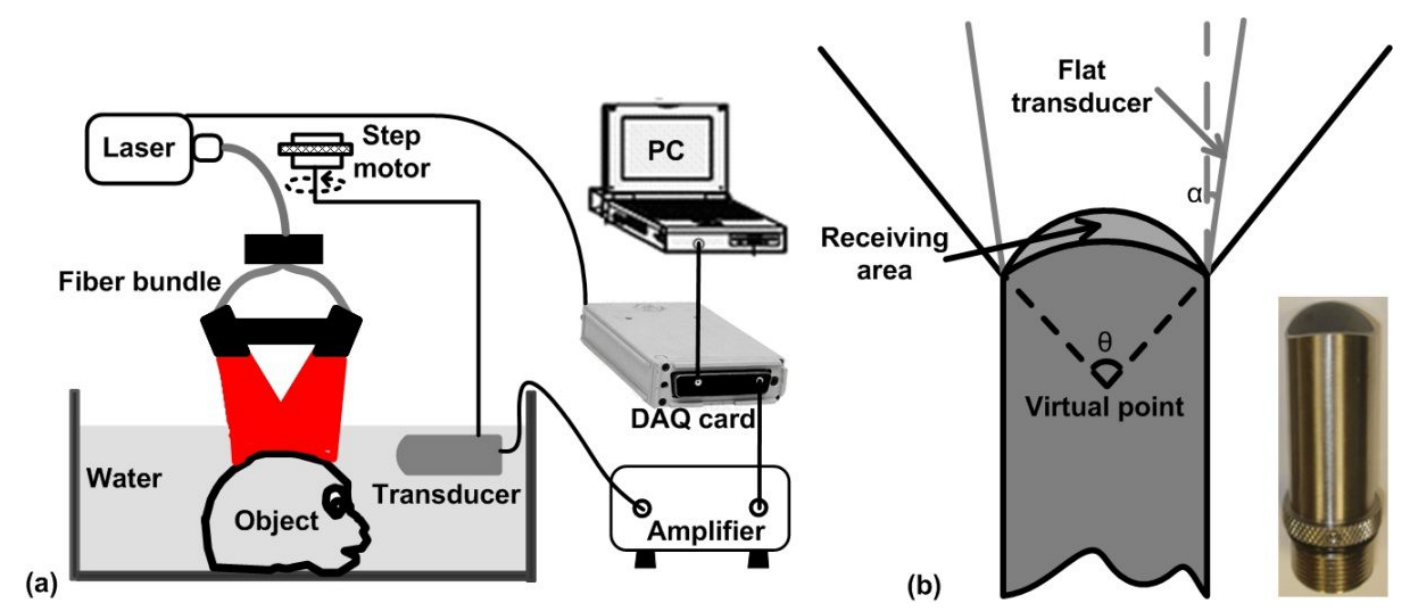

FIG. 1. (a) Schematic of the transcranial PAT system, (b) illustration of the signal acceptance mode of the virtual point detector with its photograph.

Rhesus monkey heads, obtained from the Wisconsin National Primate Research Center, were immediately immersed in 10\% formalin solution after harvest. The blood vessels were well maintained for PAT experiments, and the hair and scalp were removed. The dimensions of the head in the axial plane were about $8 \mathrm{~cm} \times 6 \mathrm{~cm}$, and the laser irradiation area was approximately $5 \mathrm{~cm} \times 5 \mathrm{~cm}$. Imaging hemoglobin oxygen saturation $\left(\mathrm{sO}_{2}\right)$ is significant to understand brain pathological information and hemodynamics. It is also invaluable for numerous medical applications, such as evaluating the effects of chemotherapy and radiotherapy, monitoring the healing process of wounds. As the first demonstration, two tubes with different $\mathrm{sO}_{2}$ were placed directly below the skull to simulate blood vessels in the brain. The plastic tubes are transparent for light to penetrate, and one tube was filled with $98 \% \mathrm{sO}_{2}$ bovine blood simulating 'artery'. The other tube was filled with $67 \% \mathrm{sO}_{2}$ bovine blood simulating 'vein'. $630 \mathrm{~nm}$ and $640 \mathrm{~nm}$ wavelengths were used to compute the $\mathrm{sO}_{2}$.

To determine the SOS and density maps of the skull for use with the time-reversal image reconstrution method, Xray CT image data were employed. The monkey skull was attached with three fiducial markers and imaged using an Xray CT scanner (Philips Healthcare, Eindhoven, The Netherlands) located at Washington University in St. Louis. Details regarding this system can be found in reference [20]. From knowledge of the CT image, the porosity map $\Phi_{k}$ was computed according to Eqn. (5). Subsequenlty, the density and SOS maps $\rho_{k}$ and $c_{k}$ were computed according to Eqns. (7) and (8). The SOS and density maps of the skull were subsequently registered to the PAT coordinate system through three fiducial markers visiable in both CT image and PAT image, which was reconstructed by a half-time algorithm [18]. 
The registered SOS and density maps were employed with the k-space time-reversal PAT image reconstruction algorithm developed by Treeby et al.

\section{RESULTS}

Fig. 2 shows PAT images of the monkey brain reconstructed by use of a back-projection algorithm with and without the cranium and dura mater. Fig. 2(a) shows the PAT image of the monkey brain without the skull, which serves as a reference image. Fig. 2(b) shows the PAT image of monkey brain with one skull, whose thickness ranged from 2-4 mm. Then we increased the skull thickness by covering a second thicker skull on the first skull, where the second skull thickness ranged from 2.5-4.5 mm. Figure 2(c) shows the PAT image of monkey brain with the two skull thicknesses. We can associate blood vessels in the different images by labeling them with the same numbers. The visible blood vessels are marked with white bold numbers, while the invisible blood vessels are marked with green numbers. In Fig. 2(b), all of the blood vessels except \# 10 are visible in the reconstructed image with one skull present. In the images corresponding to two skulls present, half of the blood vessels (\# 2, 3, 4, 5, 6, 8, 11, 12, 14, 15, and 16) can still be clearly imaged in Fig. 2(c). We quantified the mean full width at half maximum (FWHM) diameter of the reconstructed blood vessels, with two skull thicknesses and without any skull, to be $0.83 \mathrm{~mm}$ and $0.41 \mathrm{~mm}$ respectively.
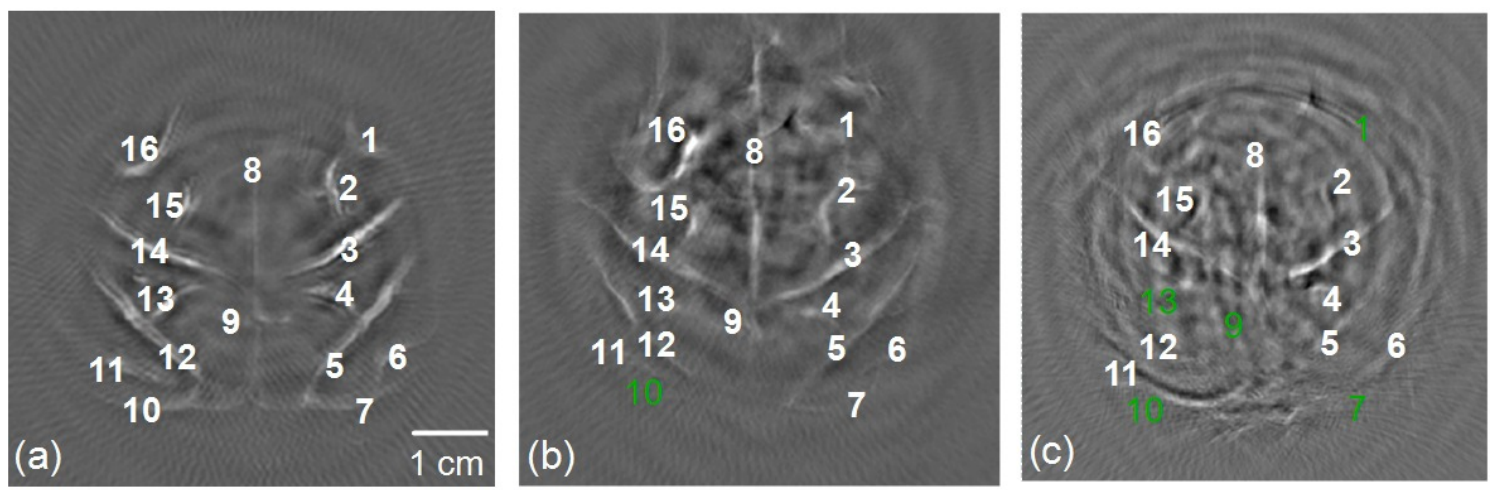

FIG. 2. PAT images of the monkey brain (a) without skull, (b) with one skull thickness, and (c) with two skull thicknesses. The visible and invisible blood vessels are marked with white and green numbers, respectively. .

The PAT image of the two tubes under the skull is shown in Fig. 3(c), corresponding to use of laser light of wavelength $630 \mathrm{~nm}$. The $\mathrm{sO}_{2}$ maps of the two tubes without and with the skull are shown in Figs. 3(a) and 3(b), where 'artery' and 'vein' are pseudocolored red and green, respectively, based on the imaged $\mathrm{sO}_{2}$ values. By comparing this result with the result measured from a gas analyzer, we determined that the computed $\mathrm{sO}_{2}$ accuracy is $\sim 8 \%$ in the PAT image corresponding to the skull present case. This suggests that transcranial PAT imaging of $\mathrm{sO}_{2}$ values is feasible, and PAT can potentially be employed for functional primate brain imaging. 

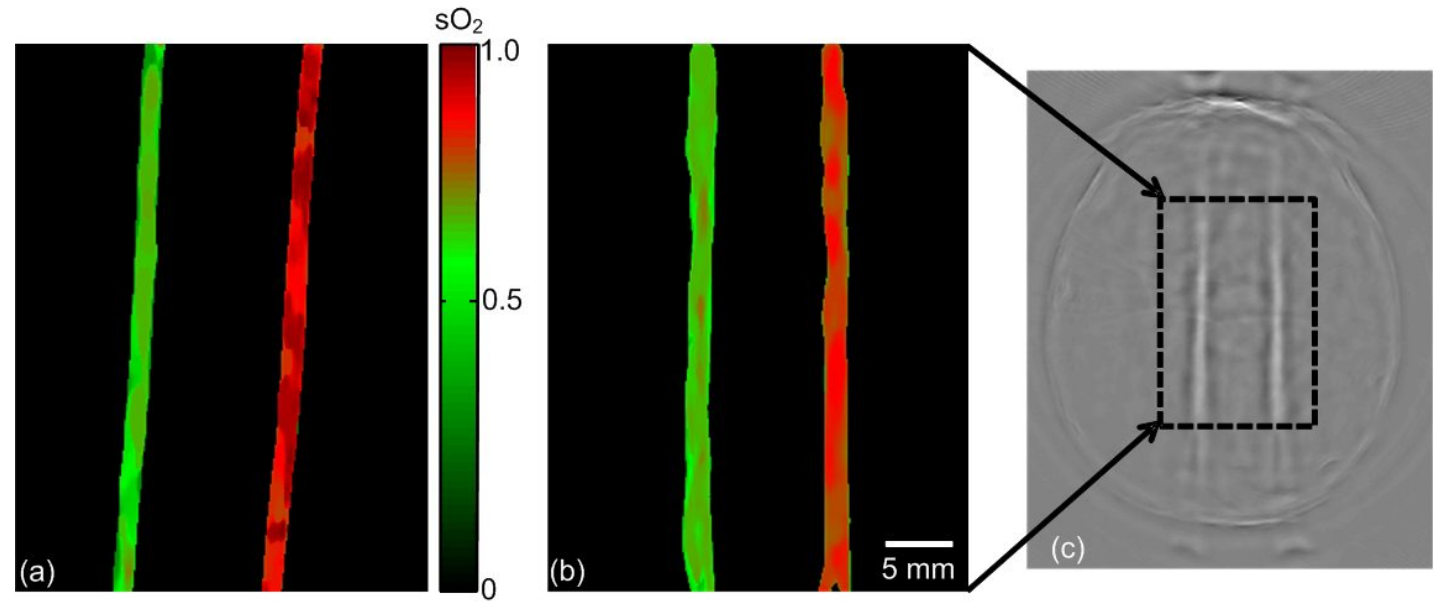

FIG. 3. PAT imaging of $\mathrm{sO}_{2}$ variation in two tubes filled with bovine blood under $630 \mathrm{~nm}$ and $640 \mathrm{~nm}$. (a) $\mathrm{sO}_{2}$ image of the two tubes within the marked region without skull, (b) $\mathrm{SO}_{2}$ image of the two tubes within the marked region with skull, (c) structural image reflecting the total hemoglobin concentration acquired at the $630 \mathrm{~nm}$ optical wavelength.

Fig. 4 shows the effectiveness of our proposed reconstructed methodology to mitigate image artifacts due to acoustic aberration caused by the skull. Fig. 4(a) is the PAT image without a skull present (the reference image) reconstructed by the back-projection algorithm. PAT images of the monkey brain with a skull present reconstructed by use of the back-projection and time-reversal algorithms are shown in Figs. 4(b) and 4(c), respectively. All images have been normalized to their maximum pixel value, and are displayed in the same grey-scale window. As can be seen, the image reconstructed by use of the time-reversal algorithm in Fig. 4(c) contains reduced artifact levels as compared to Fig. 4(b) and is much closer in appearance to the reference image. Note that the blood vessels near the skull boundary in fig. 4(c) are well compensated for and much clearer than in the image without aberration compensation [fig. 4(b)].
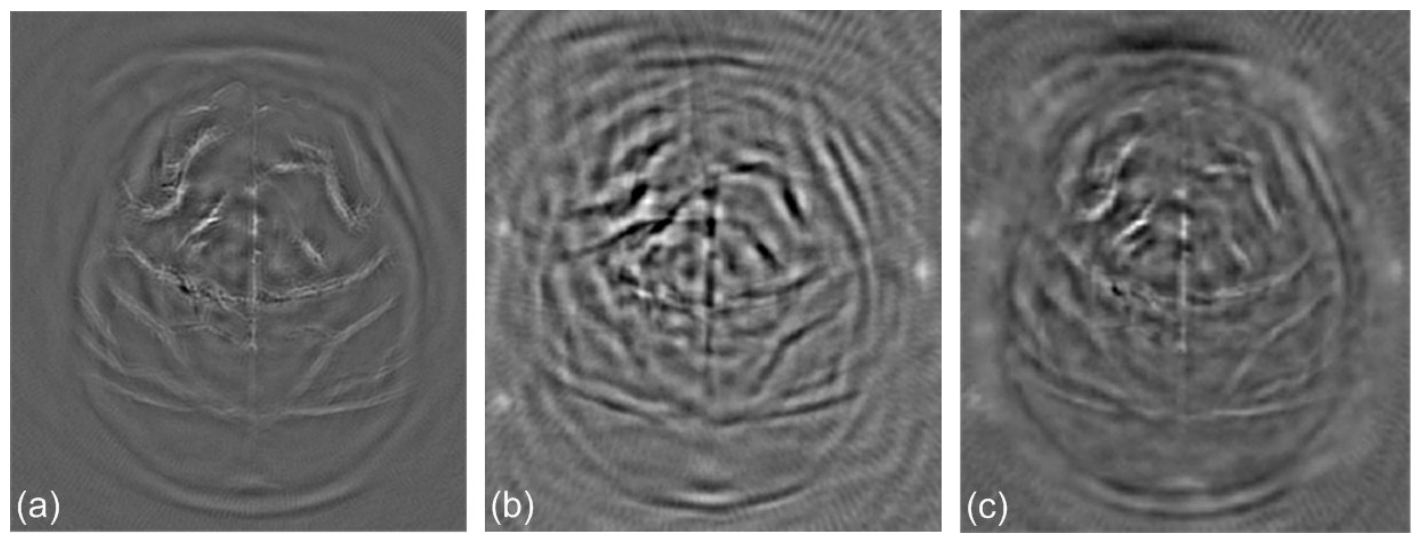

FIG. 4. Reconstructed image of the monkey brain (a) without skull using back-projection, (b) with skull present using the backprojection algorithm, (c) with skull present using time reversal algorithm.

\section{CONCLUSION}

In summary, we developed and applied virtual point detectors for transcranial PAT of the monkey brain. Compared to flat ultrasonic transducers, virtual point ultrasonic transducers greatly improve the tangential resolution, radial resolution, and SNR at the periphery. The increase in the acceptance angle of the detectors enabled us to image a larger FOV, and thus the object shapes in the reconstructed images were well preserved, even when the target was far from the scanning center. Compared to the negative-lens ultrasonic transducers [19], the virtual point ultrasonic detectors provide an improved SNR ( $\sim 15 \%$ improvement) and avoid reverberation artifacts in the reconstructed images. This salient feature allowed us to successfully accomplish PAT of a monkey brain cortex, where more blood vessels were clearly 
observed than in our previous work [11]. We also demonstrated a reconstruction methodology for transcranial PAT to mitigate skull-induced aberration in the reconstructed image. Our experimental results show that the reconstruction methodology can reduce artifact levels as compared to use of a back-projection algorithm. The results suggest that our reconstruction methodology can overcome the effects of a relatively thick skull to a great extent, and that PAT brain cortex imaging of large animals is feasible. Therefore, PAT may be potentially used for monitoring the blood-related pathological and physiological status of human brains, especially for neonatal brains before the closure of fontanelles.

This research was supported by the National Institutes of Health (grants R01 EB010049, R01 CA134539, R01 EB000712, U54 CA136398, and R01 EB008085).

\section{REFERENCES}

[1] H. F. Zhang, K. Maslov, G. Stoica, and L. V. Wang, "Functional photoacoustic microscopy for high-resolution and noninvasive in vivo imaging," Nat. Biotech. 24(7), 848-851 (2006).

[2] D. Razansky, C. Vinegoni, and V. Ntziachristos, "Multispectral photoacoustic imaging of fluorochromes in small animals," Opt. Lett. 32, 2891-2893 (2007).

[3] J. G. Laufer, D. T. Delpy, C. E. Elwell, and P. C. Beard, "Quantitative spatially resolved measurement of tissue chromophore concentrations using photoacoustic spectroscopy: application to the measurement of blood oxygenation and haemoglobin concentration," Phys. Med. Biol. 52, 141-168 (2007).

[4] L. Nie et al., "Microwave-induced thermoacoustic scanning CT for highcontrast and noninvasive breast cancer imaging," Med. Phys. 35(9), 4026-4032 (2008).

[5] L. Nie, D. Xing, D. W. Yang, L. M. Zeng, and Q. Zhou, "Detection of foreign body using fast thermoacoustic tomography with a multielement linear transducer array," Appl. Phys. Lett. 90, 174109 (2007).

[6] L. Nie, et al, "Thermoacoustic molecular tomography with magnetic nanoparticle contrast agents for targeted tumor detection,” Med. Phys. 38,4193-4198 (2010) .

[7] V. P. Zharov, E. I. Galanzha, E. V. Shashkov, N. G. Khlebtsov, and V. V. Tuchin, "In vivo photoacoustic flow cytometry for monitoring of circulating single cancer cells and contrast agents," Opt. Lett. 31, 3623-3625 (2006).

[8] X. Wang, Y. Pang, G. Ku, X. Xie, G. Stoica, and L. V. Wang, "Noninvasive laser-induced photoacoustic tomography for structural and functional in vivo imaging of the brain," Nat. Biotechnol 21(7), 803-806 (2003).

[9] S. Yang, D. Xing, Q. Zhou, L. Xiang, and Y. Lao, "Functional imaging of cerebrovascular activities in small animals using high-resolution photoacoustic tomography," Med. Phys. 34, 3294-3301 (2007).

[10]H. P. Brecht, R. Su, M. Fronheiser, S. A. Ermilov, A. Conjusteau, and A. A. Oraevsky, "Whole-body three dimensional optoacoustic tomography system for small animals," J. Biomed. Opt. 14(6), 064007-1-8 (2009).

[11] X. M. Yang and L. V. Wang, "Monkey brain cortex imaging by photoacoustic tomography," J. Biomed. Opt. 13(4), 044009-1-5 (2008).

[12] Y. Xu and L. H. V. Wang, "Rhesus monkey brain imaging through intact skull with thermoacoustic tomography," IEEE Trans. Ultrason. Ferroelect. Freq. Contr. 53, 542-548 (2006).

[13]C. H. Li, G. Ku, and L. H. V. Wang, "Negative lens concept for photoacoustic tomography," Phys. Rev. E 78(2), 021901-1-5 (2008).

[14] B. Treeby and B. Cox, "k-Wave: MATLAB toolbox for the simulation and reconstruction of photoacoustic wave fields," Journal of Biomedical Optics 15, 021314 (2010).

[15]F. J. Fry and J. E. Barger, "Acoustical properties of the human skull," The Journal of the Acoustical Society of America 63(5), 1576-1590 (1978).

[16] J.-F. Aubry, M. Tanter, et al., "Experimental demonstration of noninvasive transskull adaptive focusing based on prior computed tomography scans," The Journal of the Acoustical Society of America 113(1), 84-93 (2003).

[17] Laser Institute of America, "American national standard for safe use of lasers," ANSI Z136.1-2000, American National Standards Institute, New York (2000).

[18] M.A. Anastasio, J. Zhang, et al., "Half-time image reconstruction in thermoacoustic tomography," IEEE Transactions on Medical Imaging 24, 199-210 (2005).

[19] M. Pramanik, G. Ku, and L. V. Wang, "Tangential resolution improvement in thermoacoustic and photoacoustic tomography using a negative acoustic lens," J. Biomed. Opt. 14(2), 024028-1-5 (2009).

[20] J. P. Schlomka, E. Roessl, et al., "Experimental feasibility of multi-energy photon-counting K-edge imaging in preclinical computed tomography," Physics in Medicine and Biology 53(15), 4031 (2008) 